\title{
Estudo do padrão de rompimento e da força de desligamento dos filamentos do bisso do Limnoperna fortunei (Dunker, 1857) em diferentes tipos de substratos
}

\author{
Claudia Lauria Froes \\ CETEC "FundaçãoCentroTecnológico de Minas Gerais", claudiafroes@hotmail.com \\ Madrith Sthel Costa Duarte \\ CETEC “Fundação Centro Tecnológico de Minas Gerais", madrith4@gmail.com \\ Gislene Custódio \\ CETEC “Fundação Centro Tecnológico de Minas Gerais”, giscustodio@yahoo.com.br \\ José Roberto Tavares Branco \\ CETEC "Fundação Centro Tecnológico de Minas Gerais", jose.branco@cetec.br
}

\section{Resumo}

O Limnoperna fortunei é um molusco bivalve invasor que tem causado prejuízos a sistemas de captação de água e usinas hidroelétricas. Sua presença já foi registrada nas bacias dos rios Paraná, Paraguai, Uruguai e Lago Guaíba. No presente trabalho, estudou-se a força de desligamento e o padrão de rompimento dos filamentos do bisso do L.fortunei em três tipos distintos de substratos: cobre, vidro e teflon. O cobre provocou efeito letal em 100\% dos mexilhões testados. A maior parte dos filamentos do bisso analisados após os ensaios de tração se desprendeu dos substratos testados (vidro e teflon) por rompimento. A falha adesiva ocorreu apenas em $20 \%$ dos filamentos que estavam aderidos ao teflon. Os filamentos do bisso aderidos ao vidro e ao teflon romperam em duas regiões distintas: $X$ e Y. Quanto aos tipos de rompimento, foram observados dois padrões distintos: superfície estirada de ruptura tipo estricção e superfície plana de ruptura transversal. A região de rompimento dos filamentos do bisso parece estar relacionada com as características estruturais das regiões Xe Y, não com o tipo de substrato. O substrato parece influenciar os tipos de rompimento e a força de desligamento. Esta apresentou maior valor para os mexilhões aderidos ao vidro em relação ao teflon.

Palavras chave: Limnoperna fortunei, Bisso, Mexilhão dourado, Padrão de rompimento, Força de desligamento

\begin{abstract}
Study of the pattern of disruption and detachment force of the byssus filaments Limnoperna fortunei (Dunker, 1857) in different types of substrates. The Limnoperna fortunei is a freshwater bivalve invader that has caused damage to water catchment systems and hydroelectric plants. His presence has already been recorded in the basins of the rivers Parana, Paraguay, Uruguay and Guaíba Lake. In this present work, was conducted a study about the detachment force and pattern of disruption of the byssus filaments on different substrates. Three types of materials were selected (copper, teflon and glass). Copper had a lethal effect on a $100 \%$ of the tested mussels. The adhesive failure occurred only in the Teflon. The byssus filaments adhered to glass and teflon disrupted in two distinct regions: $X$ (next to the body) and $Y$ (away/far from the body). Also there were observed, on these filaments, two distinct types of disruption: stretched surface rupture and flat surface transverse rupture. The region of disruption of the filaments of byssus seems related to the structural characteristics of regions $X$ (next to the body) e $Y$ (away/far from the body) and not with the type of substrate. The substrate appears to influence the kinds of disruption and detachment force. This presents a greater value for the mussels attached to glass compared to Teflon.
\end{abstract}

Key words: Limnoperna fortunei, Byssus, Disrupture pattern, Golden mussel, Detachment force. 


\section{Introdução}

O Limnoperna fortunei (Dunker, 1857) é um molusco bivalve invasor conhecido como mexilhão dourado, adapta-se facilmente a diferentes tipos de habitats (Darrigran; Pastorino, 1995). Dentre os impactos ambientais e econômicos causados pelo L. fortunei destacam-se o efeito sobre a comunidade fitoplanctônica e a ciclagem de nutrientes, a competição com outros animais nativos que se alimentam de partículas em suspensão, a obstrução das tubulações de estações de tratamento, reservatórios e barragens, além de outras estruturas que são muito prejudicadas pelo crescimento descontrolado do mexilhão dourado (Darrigran \& Pastorino, 1995; Darrigran \& Drago, 2000).

O Limnoperna fortunei produz fios de bisso que possibilitam a macroaglomeração sobre substratos duros (Mansur \& Pereira, 2006). Os mexilhões se fixam aos substratos, secretando, sobre suas superfícies, os filamentos do bisso, produzidos a partir de glândulas localizadas no pé do organismo. Esses filamentos constituem-se, principalmente, de colágeno, mas contêm polifenólicos hidrófilos, que são proteínas adesivas, entre as quais ocorre ligação cruzada por reação de oxidaçãoredução, envolvendo um catalisador de polifenol-oxidase (Brady et al., 2001).

O bisso produzido pelo $L$. fortunei permite sua aderência a uma grande variedade de substratos rígidos. A força de desligamento dos mexilhões ao substrato pode estar relacionada a uma variedade de características próprias ao tipo de substrato incluindo a energia livre de superfície. A literatura sugere uma relação entre força de desligamento e energia livre de superfície, indicando-se que, quanto mais baixa a energia livre de superfície do substrato, menor a força de desligamento do L. fortunei, sendo que o organismo se liga, fortemente, às superfícies polares e, fracamente, às superfícies não polares (Matsui et al., 2001). Com isso, o padrão de rompimento dos filamentos do bisso também poderá variar de acordo com as características de cada material já que a ligação do bisso aos materiais tende a ser menor em substratos com baixa densidade de pontes de hidrogênio e energia livre de superfície, como é o caso do teflon, se destacando assim mais comumente por falhas adesivas. Por outro lado, o bisso tende a se aderir fortemente em superfícies com alta densidade de pontes de hidrogênio e energia superficial, como é o caso do cobre e do vidro. Neste tipo de superfície, verifica-se a tendência de que o molusco se desligue preponderantemente com a quebra dos fios de bissos (Matsui et al., 2001).

Diversas pesquisas têm sido realizadas no intuito de investigar materiais e revestimentos, com base na engenharia de superfícies, com potencial de utilização no controle das incrustações do mexilhão dourado em plantas industriais, principalmente em unidades geradoras de energia (Bergmann, et al., 2010).

Este trabalho tem como objetivo compreender os padrões de rompimento dos filamentos do bisso em três tipos de materiais com diferentes naturezas químicas e diferentes valores de energia livre de superfície como vidro, cobre e teflon, obtendo-se assim uma melhor compreensão das propriedades físicas e químicas da superfície dos substratos que possam influenciar ou não a fixação do L. fortunei e, consequentemente, auxiliar no desenvolvimento de tecnologias para o controle desta espécie invasora.

\section{Material e Métodos}

\subsection{Coleta e preparo dos indivíduos}

Os exemplares do Limnoperma fortunei foram coletados nos estados de São Paulo (reservatórios de Jupiá e Porto Primavera) e Rio Grande do Sul (rio Guaíba, Porto Alegre), e posteriormente mantidos em laboratório. Por se tratar de uma espécie invasora, foram seguidas as normas de biossegurança estabelecidas (Campos et al., 2003).

Os mexilhões foram colocados em aquários de 20L, em média 500 indivíduos por aquário, com água declorada e aeração contínua. A temperatura média da água dos aquários foi de $25^{\circ} \mathrm{C}$. A água dos aquários foi trocada três vezes por semana, onde se retirava metade da água de cada aquário, renovando a mesma gradativamente. Organismos mortos eram descartados diariamente.

Para alimentação diária foi utilizado composto à base de alga Selenastrum capricornutum (Printz, 1914) e ração de peixe com teor protéico maior que $40 \%$ dissolvido em água destilada. A quantidade de alimento foi definida através de testes realizados em laboratório levando em consideração a densidade de mexilhões por aquário, onde foi fornecido $100 \mathrm{ml}$ do alimento a base de alga e $50 \mathrm{ml}$ da ração de peixe com teor protéico maior que 40\%.

Foram selecionados para os ensaios os mexilhões bissados, com concha intacta e que respondiam a estímulos externos. O tamanho médio dos mexilhões foi de $2,0 \mathrm{~cm}$.

Foram selecionados 100 indivíduos por material testado. Estes foram transferidos para 3 outros aquários testes com medidas: $58 \times 27 \times 29 \mathrm{~cm}$. A base dos aquários foi revestida com placas de $8 \times 3 \mathrm{~cm}$ de cada material testado, perfazendo uma área de $1,7 \mathrm{~m}^{2}$. Foi mantida uma proporção de 2 litros de água declorada para cada 10 organismos. O primeiro aquário teve sua base recoberta com placas de cobre, o segundo por placas vidro e o terceiro por placas de teflon. Os indivíduos permaneceram nestes aquários testes por 45 dias.

O experimento foi mantido sob aeração e fluxo de água contínuo. Para a geração do fluxo de água foi utilizada uma bomba d'água submersível para aquário (Submersible pump) da marca Resun SP-980, com velocidade do fluxo de 550 L/H. Esta bomba foi colocada na 
porção superior do aquário, no limite com a superfície. 0 fluxo de água foi utilizado a fim de aproximar o teste da realidade ambiental dos organismos.

\subsection{Seleção e preparo dos substratos}

Os materiais foram selecionados levando em conta as diferenças em suas propriedades, como energia livre de superfície. Foram escolhidos três tipos de materiais:

a) Polímero: politetrafluoretileno - PTFE, conhecido como teflon - com energia livre de superfície de $19,5 \mathrm{~mJ} / \mathrm{m}^{2}$ (Faria, 2006).

b) Cerâmico: vidro - com energia livre de superfície de $160,3 \mathrm{~mJ} / \mathrm{m}^{2}$ (Faria, 2006).

c) Metálico: cobre - com energia livre de superfície de $177 \mathrm{~mJ} / \mathrm{m}^{2}$ (Müller, 2002).

As amostras citadas receberam tratamento padrão adotado por Faria (2006), que consiste em lavagem em solução de surfactante $2 \%$ e enxágue em banho ultrassônico com acetona. Antes da colocação em aquários para o teste, cada material recebeu limpeza com álcool 70\% e enxágue em água destilada.

\subsection{Ensaios de tração}

Análise da força de desligamento do mexilhão dourado. Foi realizado levantamento sobre máquina de testes capaz de realizar medidas de tração e compressão com carga máxima de avaliação em $3 \mathrm{Kgf}$ que atendesse à necessidade de ensaio para o estudo de força de desligamento de organismos bivalves. Devido ao alto custo de aquisição e adequação às normas de segurança, foram seguidos procedimentos segundo Campos et al, 2003, para adaptação de equipamento de análise de tração.

O equipamento é constituído por uma bandeja móvel com velocidade controlável por um inversor de freqüência, transdutor de força acoplado ao sistema com instrumentação eletrônica para indicação de pesagem, garra para fixação do organismo e fio metálico para acoplagem garra-transdutor de força, Figura 1.

Garra apropriada para fixação do organismo é ligada ao transdutor de força por fio metálico. Os transdutores de força selecionados são da marca HBM, modelo PW4KC3, e apresentam capacidade máxima de leitura nas faixas de 2,94N, 4,90N e 29,42N. Os transdutores de força foram certificados no Laboratório Isaac Newton do Setor de Teste Físicos/CETEC, por meio de calibração a compressão segundo ASTM E 74-06 e Norma do Sistema de Qualidade CETEC NSQC 1102.

O software foi desenvolvido no Setor de Materiais Óticos e Eletrônicos/CETEC para aquisição de dados força em relação a tempo.

Para os ensaios com o organismo, as placas de teflon, vidro e cobre contendo exemplares de mexilhão dourado aderidos por 45 dias foram retiradas dos aquários. Estas foram secas com papel toalha e fixadas na bandeja do equipamento de tração. Os filamentos de bissos tracionados aderidos às placas de cada material teste foram analisados em seguida através de microscopia óptica.

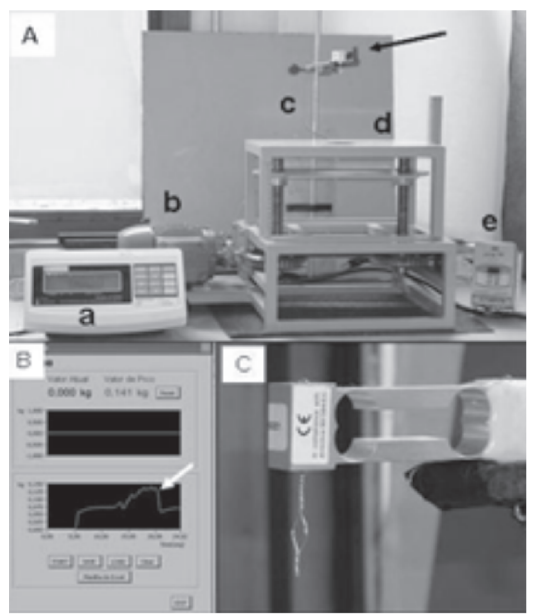

Figura 1: Equipamento de tração para medida da força de desligamento do Limnoperna fortunei: a- Indicador de pesagem; $b$-motor; c-suporte para fixação do transdutor de força (seta contínua); d- suporte com bandeja móvel; einversor de freqüência para controle de velocidade da bandeja móvel. B- Registro gráfico da força durante o desligamento do organismo ao material e o pico no momento do arrancamento (seta contínua). C- Detalhe do transdutor de força do equipamento de tração.

A estrutura metálica é constituída de suporte fixo onde apenas a bandeja é movimentada a velocidade controlada. A bandeja permite a fixação do material em estudo com o organismo aderido.

\subsection{Análise dos bissos rompidos (padrão de rompimento)} As regiões de possível rompimento foram definidas a partir de observações morfológicas e características dos filamentos do bisso encontradas na literatura. Para este estudo foram consideradas duas regiões de possível rompimento: região distante do corpo, região do filamento bisso com comprimento de até $400 \mu \mathrm{m}$ a partir da placa bissal (Y) e região próxima ao corpo (X), comprimento superior a $400 \mu \mathrm{m}$ (Figura 2). O tipo de rompimento foi definido durante a observação microscópica dos filamentos.

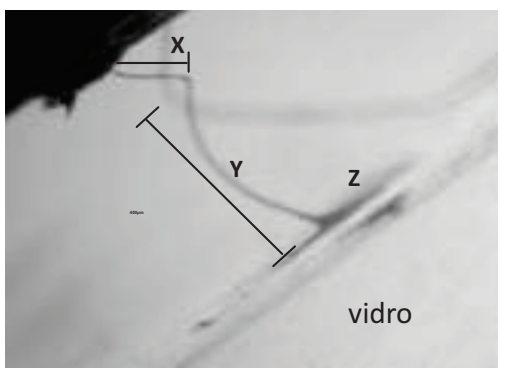

Figura 2: Fotomicrografia de um filamento de bisso aderido a uma lâmina de vidro (magnitude 100x). $X$ - Região próxima ao corpo; Y - Região distante do corpo; Z - Placa bissal aderida ao vidro. 


\subsection{Análise estatística}

Foram analisados 100 filamentos de bisso tracionados para cada material testado, independente do animal que o produziu, com o intuito de se conhecer o comportamento mecânico destes filamentos quando submetidos a forças de tração.

A análise estatística dos resultados do padrão de rompimento e da força de desligamento dos filamentos do bisso aos materiais testados foi realizada através de testes não paramétricos com um baixo valor de $n$. Utilizou-se o teste $U$ de Mann-Whitney para as comparações entre dois grupos independentes e correlação de Spearman entre grupos. Todas as análises foram feitas utilizando nível de significância $a \leqslant 0,05$. O programa estatístico utilizado foi o Statistica program for windows, version 7, Statsoft, Inc. EUA. 1984-2004.

\section{Resultados}

Entre os materiais testados nos ensaios de tração, somente o cobre demonstrou ser tóxico aos mexilhões (100\% de efeito letal sobre os organismos testados).

\subsection{Força de desligamento}

Durante os ensaios de tração, os filamentos de bissos tracionados sofreram uma deformação média no estiramento entre $20 \%$ e 50\% linearmente à tensão aplicada até o momento da ruptura (figura 3 ).

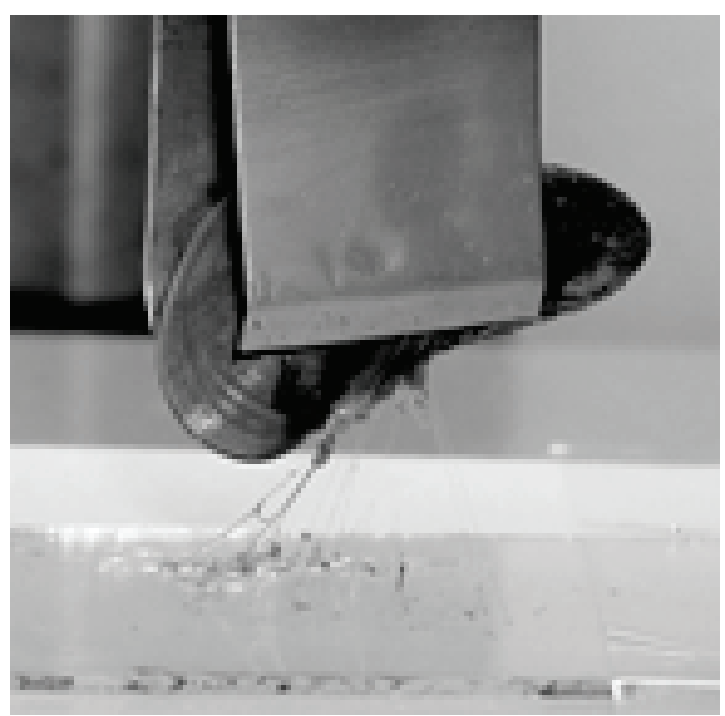

Figura 3- Filamentos do bisso de um exemplar de mexilhão dourado aderido a uma lâmina de vidro sendo estirados quando submetidos ao ensaio de tração.

As informações de força de desligamento para os materiais segundo análise estatística descritiva se encontram na tabela 1.

\begin{tabular}{ccccc} 
Substrato & \multicolumn{2}{c}{$\begin{array}{c}\text { Força de } \\
\text { desligamento (N) }\end{array}$} & \multicolumn{2}{c|}{$\begin{array}{c}\text { Número de bissos } \\
\text { secretados }\end{array}$} \\
\hline Vidro & 2,53 & 1,64 & 17,89 & 9,69 \\
Teflon & 1,77 & 0,91 & 15,30 & 1,27 \\
\hline
\end{tabular}

Tabela 1: Resultados de teste de tração em vidro e teflon e números de bissos secretados.

No teste de normalidade (distribution fitting), verificou-se assimetria para os dados de força de desligamento dos organismos em Teflon, $p=0,000$, e em vidro, $p=0,007$ ( $p<0,05$ diferença significativa). Para os dados de filamentos de bissos, observou-se simetria em relação ao teste de normalidade tanto para Teflon, $p=$ 0,168 , quanto para vidro, $p=0,063$ ( $p>0,05$ não há diferença significativa). Os resultados estatísticos podem ser visualizados na figura 4. De acordo com análise estatística, houve diferença significativa em relação à força de desligamento entre os materiais testados onde os filamentos aderidos ao vidro apresentaram maior valor.

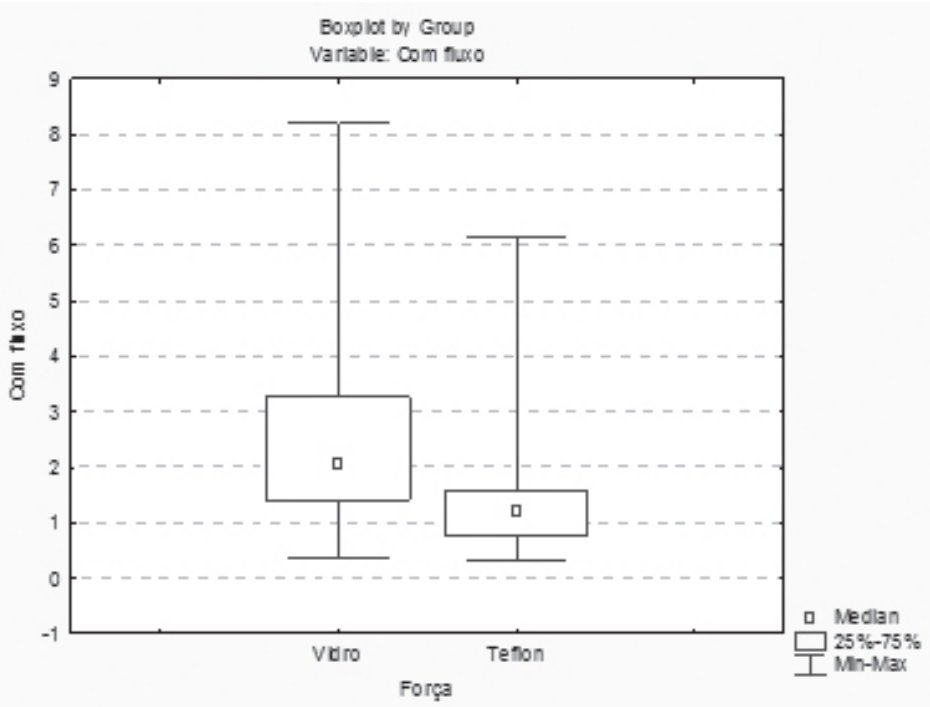

Figura 4: Comparação entre força de desligamento em materiais, vidro e teflon.

\subsection{Padrão de rompimento}

No teflon, $80 \%$ dos filamentos do bisso analisados se desprenderam do substrato por rompimento. $O$ restante, $20 \%$, se desprendeu do substrato através de falha adesiva, ou seja, descolamento da placa bissal do substrato (Figura 5). No vidro, $100 \%$ dos filamentos analisados se desprenderam dos substratos testados por rompimento. Os filamentos dos bissos rompidos analisados romperam em duas regiões distintas, $X$, próxima ao corpo $(>400 \mu \mathrm{m})$ (Figura 6 A), e Y, distante do corpo $(<400 \mu \mathrm{m}$ ) (Figura 6 B). 


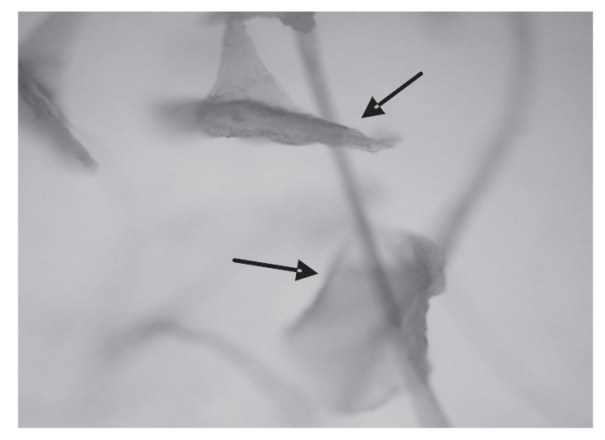

Figura 5: Foto micrografia (magnitude 400x) de duas placas bissais (setas) que se descolaram do substrato teflon durante os ensaios de tração.
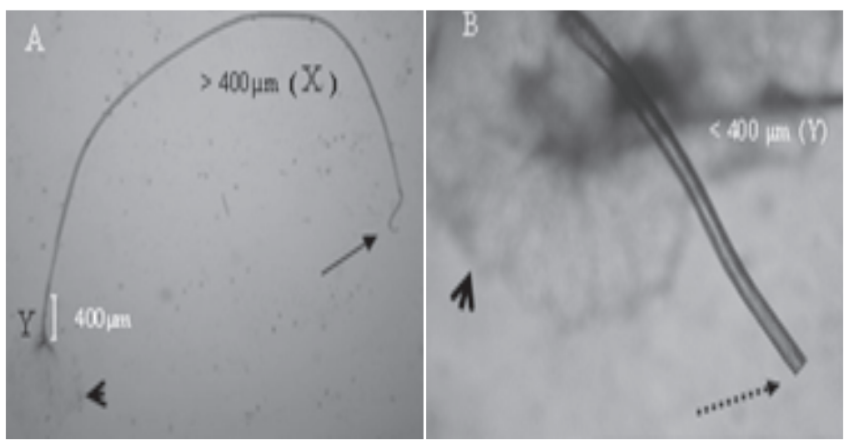

Figura 6: Fotomicrografia de filamentos do bisso do mexilhão Limnoperna fortunei rompidos após ensaio de tração. A (magnitude 100x) - Filamentos do bisso aderidos a um substrato através da placa bissal ou placa adesiva (cabeça de seta) que apresentaram rompimento na região $X$ (seta contínua). B (magnitude 400x) - Filamento do bisso com rompimento na região $Y$ (seta tracejada) e placa bissal (cabeça de seta).

O resultado da ocorrência das regiões de rompimento dos filamentos do bisso do Limnoperna fortunei está apresentado na Figura 7. Foi observada maior ocorrência de rompimentos na região $X$ dos filamentos, tanto para o vidro (95\%) quanto para o teflon (80\%).

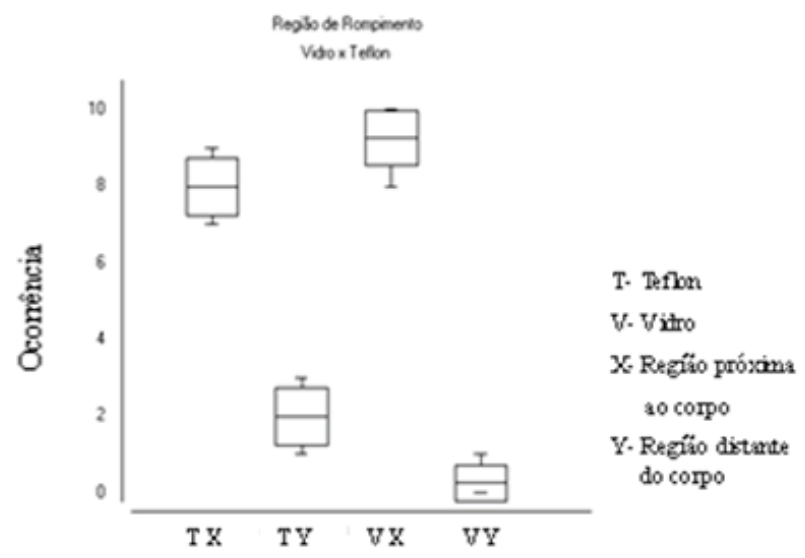

Figura 7: Ocorrência das regiões de rompimento dos filamentos do bisso do Limnoperna fortunei para o vidro e para oteflon.
Os filamentos do bisso tracionados apresentaram também dois tipos de rompimento: superfície plana de ruptura transversal (Figura 8 A e B) e superfície estirada de ruptura tipo estricção (Figura $8 \mathrm{Ce}$ D).

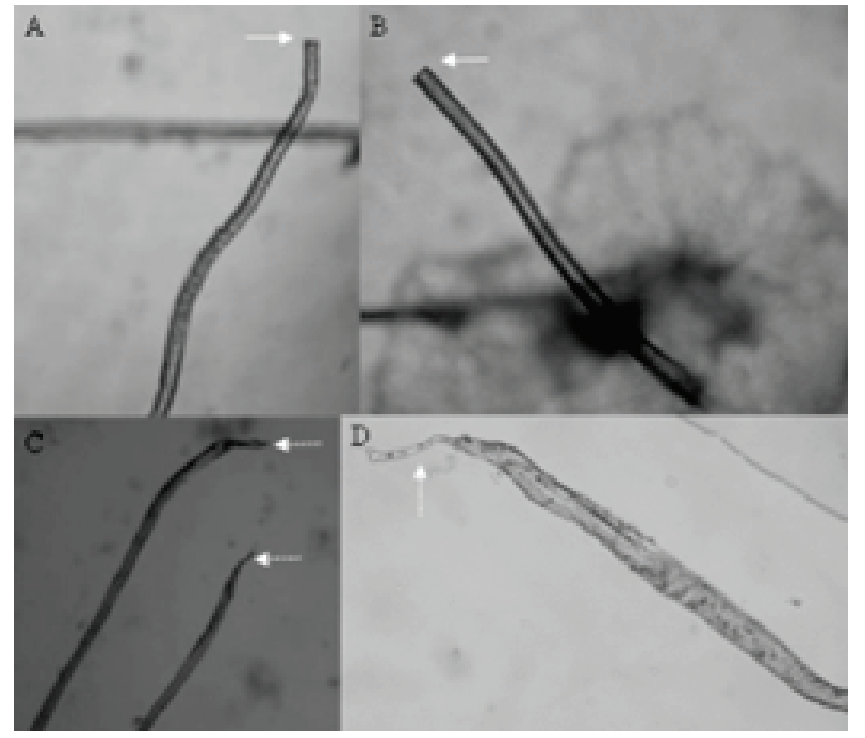

Figura 8: Fotomicrografia de filamentos de bisso (magnitude 400x) após ensaios de tração, com diferentes tipos de rompimento. A e B - Tipos de rompimento: superfície plana de ruptura transversal (setas). C e D - Tipos de rompimento: superfície estirada de ruptura tipo estriç̧ão(setas).

A Figura 9 mostra o resultado da ocorrência dos dois tipos de rompimento dos filamentos de bisso para o vidro e o teflon. Foi observada uma prevalência do tipo superfície plana de ruptura transversal para os filamentos que estavam aderidos ao vidro (56\%) quando comparados ao teflon (30\%). Quanto ao rompimento superfície estirada de ruptura tipo estricção, observou-se uma maior ocorrência no teflon (70\%) em relação ao vidro (44\%).

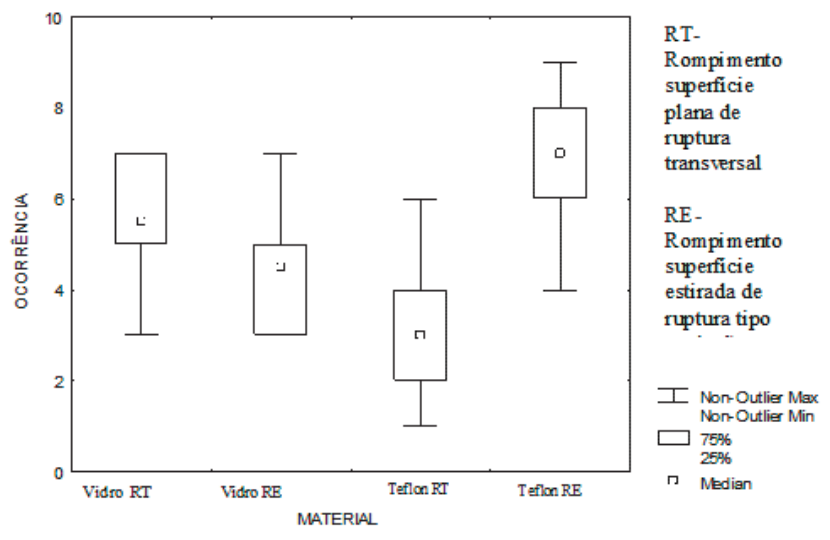

Figura 9: Ocorrência dos tipos de rompimento (superfície plana de ruptura transversal e superfície estirada de ruptura tipo estricção) dos filamentos de bisso do Limnoperna fortunei para o vidro e para o teflon. 


\section{4-Discussão}

Segundo Matsui et al (2001), a força média de desligamento para o material vidro é de $1,49 \mathrm{~N}$ enquanto para o teflon é de $0,12 \mathrm{~N}$. Segundo Faria (2005), a força média de desligamento no vidro é de $0,49 \mathrm{~N}$ e para o teflon é de $0,19 \mathrm{~N}$. Os valores mostram, comparativamente com dados deste trabalho citado na tabela 1, que os filamentos do bisso tendem a se aderir fortemente a superfícies com alta energia livre superficial gerando uma maior força de desligamento.

Nota-se que a diferença entre os valores de força de desligamento dos organismos para os mesmos tipos de substratos testados encontrados por Matsui et al (2001) e Faria (2005) e dados da tabela 1 pode estar relacionada a variáveis utilizadas no ensaio, como: tempo de adesão dos organismos, presença de fluxo de água, estado do substrato e equipamento de testes.

Nos ensaios realizados por Matsui et al (2001), mexilhões coletados em rio e mantidos em laboratório para nova adesão em sistema estático aos materiais de estudo por um período de 7 dias apresentavam tamanho de concha entre $2-3 \mathrm{~cm}$ saudáveis e respondendo a estímulos externos. $O$ equipamento utilizado para ensaios de tração foi o EZ Test 20N, Shimadzu Corporation, Kyoto, Japão.

No trabalho de Faria (2005), as condições de ensaio seguidas foram semelhantes ao de Matsui (2001), onde, segundo Faria (2005), os organismos foram presos aos materiais testados por um barbante para forçar a fixação dos organismos. O teste teve duração de 7 dias, durante os quais as medições da força de desligamento foram realizadas manualmente com aparato envolvendo fio metálico, garra e balança.

A força de desligamento observada neste trabalho, muito superior ao trabalho de Matsui (2001) para teflon, pode estar relacionada ao tempo de adesão e fluxo de água. Apesar da quantidade de fios de bissos ser semelhante, sugere-se que a maior força observada pode dever-se a um maior número de interações de hidrogênio entre placa bissal e substrato.

Maior força de desligamento também se observa no presente trabalho para o vidro em relação ao estudo de Matsui (2001) apesar de um menor valor de número de filamentos de bissos. Estes dados sugerem que, assim como para o teflon, a adesão mais forte via ligações químicas independe do número de filamentos secretados. O método dinâmico, com fluxo de água, pode ter colaborado para melhor adesão do organismo neste material.

Em materiais com alta energia livre de superfície, verifica-se a tendência de que o molusco se desligue preponderantemente com o rompimento dos fios dos bissos (Matsui et a.l, 2001). Este fato foi observado nos ensaios realizados nos quais os filamentos do bisso aderidos ao vidro, material com alta energia livre de superfície, romperam-se após os ensaios de tração.

De acordo com Matsui et al. (2001), a ligação dos filamentos do bisso tende a ser menor em substratos com baixa energia livre de superfície, ocasiões em que falhas adesivas tornam o desprendimento mais comum. Este fato explica a ocorrência da falha adesiva somente nos filamentos que estavam aderidos ao teflon. Devido à baixa energia livre superficial deste material, era esperado um número mais expressivo de falhas adesivas. Porém, os experimentos foram realizados sobre a influência de um fluxo contínuo de água, o que pode ter induzido os mexilhões a produzirem filamentos de bisso mais resistentes. De acordo com Carrington (2002), os mexilhões podem detectar e responder as mudanças de fluxo da água em seu ambiente, produzindo um bisso mais forte que resista a elas.

A força de desligamento pode estar associada a ocorrência da falha adesiva observada nos filamentos que estavam aderidos ao teflon, o que não aconteceu para o vidro.

De acordo com a figura 7, a grande maioria dos filamentos dos bissos analisados, tanto no vidro quanto no teflon, romperam na região $\mathrm{X}$, próximo ao corpo. Quando comparamos os filamentos com rompimento na região $X$ que estavam aderidos ao vidro com os que estavam aderidos ao teflon, o valor de $\mathrm{p}$ foi igual a 1,000. Já para a comparação entre os filamentos com rompimento na região $Y$, para esses mesmos materiais o valor de $p$ foi igual a 0,658. Conclui-se, então, de acordo com a análise estatística, que não ocorreu diferença significativa de um material para o outro em relação à região de rompimento dos filamentos do bisso, onde esta parece estar relacionada com as características estruturais das regiões Xe Y e não com o tipo de substrato.

Foi observado nos filamentos do bisso com rompimento na região $X$ que estavam aderidos ao vidro e ao teflon que, nesta região do filamento, ocorre estiramento antes do rompimento. Este fato está de acordo com Wiegemann (2005), que afirma ser a região proximal (X) mais elástica que a distal (Y), sofrendo estiramento quando submetida a forças de tração.

Já os filamentos tracionados, que romperam na região $Y$, no vidro e no teflon, não sofreram estiramento antes do rompimento. Isto mostra que as duas regiões apresentam comportamentos mecânicos diferentes: uma sofre estiramento antes do rompimento e a outra não. Fato que pode estar ligado às diferenças estruturais de cada região do filamento, onde, segundo Carrington (2002), a região $Y$, distante do corpo, é mais dura, formada por fibras de colágeno densamente empacotadas, mais fortes e menos extensas do que a região proximal considerada um material compósito, com fibras de colágeno reforçando uma matriz protéica macia.

Ao se comparar os dois materiais em relação ao tipo de 
rompimento, pode-se inferir, após análise estatística, que houve diferença significativa de um material para o outro em relação ao tipo de rompimento. No vidro, a quantidade de filamentos com rompimento do tipo superfície plana de ruptura transversal foi maior que no teflon $(p=0,003)$. Já a quantidade de filamentos de bissos com rompimento do tipo superfície estirada de ruptura tipo estricção foi maior no teflon do que no vidro $(p=0,003)$.

O tipo de rompimento variou de um material para o outro. Esta variação pode ser devida a valores distintos da energia livre de superfície entre os dois materiais testados, fato que pode ter influenciado no processo de formação dos filamentos do bisso, podendo assim alterar as suas propriedades mecânicas. Esta alteração nas propriedades mecânicas dos filamentos pode acarretar diferenças em relação ao tipo de rompimento.

O comportamento da região $\mathrm{X}$ em relação ao tipo de rompimento foi diferente no vidro e no teflon. Este fato pode ser devido à alta energia livre de superfície do vidro, que pode ter influenciado o processo de formação dos filamentos do bisso. Em relação ao tipo de rompimento, a região $X$ parece sofrer influência do substrato, diferentemente do observado na região $Y$.

\section{Conclusão}

Conclui-se que o cobre provocou efeito letal em 100\% dos mexilhões testados.

A força de desligamento maior foi observada para o vidro em relação ao teflon, no qual se pode observar presença de falha adesiva. Em materiais com alta energia livre de superfície, verifica-se a tendência de que o molusco se desligue preponderantemente com o rompimento dos fios dos bissos.

Em relação ao padrão de rompimento, os filamentos do bisso romperam em duas regiões distintas ( $\mathrm{X}$ e $\mathrm{Y}$ ). Houve uma predominância de rompimento na região $X$ dos filamentos de bisso que estavam aderidos ao vidro e ao teflon em relação ao rompimento $Y$ destes filamentos para os mesmos substratos. A região de rompimento, $\mathrm{X}$ ou $\mathrm{Y}$, parece estar relacionada com as suas características estruturais e não com o tipo de substrato.

Foram observados dois tipos de rompimento distintos nos filamentos que estavam aderidos ao vidro e ao teflon: superfície estirada de ruptura tipo estricção e superfície plana de ruptura transversal.

O tipo de rompimento variou de um material para o outro. Esta variação pode ser devida a valores distintos da energia livre de superfície entre os dois materiais testados, fato que pode ter influenciado no processo de formação dos filamentos do bisso, podendo assim alterar as suas propriedades mecânicas. Esta alteração nas propriedades mecânicas dos filamentos pode acarretar em diferenças em relação ao tipo de rompimento.

\section{Agradecimentos}

Ao CETEC e IEAPM.

\section{Referências Bibliográficas}

BERGMANN, C.P; MANSUR, M.C.D; PEREIRA, D; BERGONCI, P.E.A; SANTOS, C.P. dos; BASEGIO, T; VICENZI, J; SANTOS, S.C.A.. Avaliação de materiais e revestimentos para controle de incrustações do mexilhão dourado Limnoperna fortunei (Dunker, 1857). Revista Biotemas. Florianópolis, v.23, n.02, p.87-100, 2010.

BRADY, R. F. JR. A fracture mechanical analysis of fouling release from nontoxicantifouling coatings. Progress in Organic Coatings, Washington, v. 43, p. 188-192, 2001.

CAMPOS, M.C.S.; RUCKERT, G.; ROLLA, M.E. Nota sobre procedimento de segurança no cultivo e manutenção de Limnoperna fortunei (Dunker,1857) em laboratório. In: IX Congresso Brasileiro de Liminologia, Juiz de Fora, 2003.

CARRINGTON, E. The ecomechanics of mussel attachment: from molecules to ecosystems. In: ANNUAL MEETING OF THE SOCIETY FOR COMPARTIVE AND INTEGRATIVE BIOLOGY, 42., 2002, Anaheim. Anais... Anaheim: SICB, 2002.p. 846-852.

DARRIGRAN, G.; DRAGO, I. E. Invasion of the exotic freshwater mussel Limnoperna fortunei (Dunker, 1876) (Bivalvia: Mytilidae) in South America. Nautilus, Sanibel, v. 114, n. 2, p. 69-73, 2000.

DARRIGRAN, G.; PASTORINO, G. The recent introduction of a freshwater Asiatic bivalve, Limnoperna fortunei (Mytilidae) into South America. Veliger, Santa Barbara, v. 38, n. 2, p. 171-175, 1995.

FARIA, E. A. Caracterização de superfícies antiincrustantes para o Limnoperna fortunei- mexilhão dourado. 2005. 58 f. Dissertação (Mestrado em Engenharia de Materiais) -Universidade Federal de Ouro Preto.

FARIA, E. A.; BRANCO, J.R. T.; CAMPOS, M.C. S.; OLIVEIRA, M. D.; ROLLA, M. E.. Estudo das características antiincrustantes de materiais. Revista da Escola de Minas, Ouro Preto, v.59, n.01, p.233-238, 2006.

MANSUR, M. C. D.; PEREIRA, D. Bivalves límnicos da bacia do rio dos Sinos, Rio Grande do Sul, Brasil (Bivalvia, Unionoida, Veneroida e Mytiloida). Revista Brasileira de Zoologia, Curitiba, v.23, n 4, p. 1123-1147, 2006. 
MATSUI, Y.; NAGAYA, K.; YUASA, A.; NARUTO, H.; YAMAMOTO, H.; OHKAWA, K.; MAGARA, Y. Attachment strength of Limnoperna fortunei on substrates, and their surface properties. Biofouling, Londres, v. 17, n. 1, p. 2939, 2001.

MÜLLER, A. Solidificação e análise térmica dos metais. $1^{\mathrm{a}}$ ed. Porto Alegre. Editora UFRGS, 2002.278 p.

VACCARO, E.; WAITE, J. H. Yield and post-yield behavior of mussel byssal thread: a self-healing biomolecular material. Biomacromolecules, Santa Barbara, v. 2, n. 3, p. 906-911, 2000.

WIEGEMANN, M. Adhesion in blue mussels (Mytilus edulis) and barnacles (genus Balanus): Mechanisms and technical applications. Aquatic Sciences, Bremen, v. 67, p. 166-176, 2005. 